\title{
HERITABLE CHARACERS OF MAIZE:
}

\section{CRINKLY LEAF'}

\author{
R. A. EMERSon \\ College of Agriculture, Cornell University, Ithaca, N. Y.
}

$I^{N}$ N 1910 a strain of dent corn obtained at the National Corn Exposition held at Omaha was crossed with a strain of flint corn obtained from the Department of Agronomy of the University of Nebraska. The $F_{1}$ plants of this cross were normal and no abnormalities had been observed in the parent strains. But since the latter had not been subjected to self-pollination, there is no assurance that one or other of them did not have in it the character to be described here. In the $\mathrm{F}_{2}$ generation of this cross there occurred a type of plant that has later been called "crinkly."

Crinkly is a semi-dwarf type of plant, usually about two-thirds the height of normal plants of the same cultures (Fig. 24). The tassels of crinkly are relatively short and compact and not infrequently bear numerous seeds. Sometimes only a few such seeds develop but of ten a part of the central spike of the tassel is more or less earlike.

The upper leaf blades of crinkly are relatively short and broad, are usually much crinkled and of ten have prominent lobes near the base (Fig. 25). All of these characteristics are so variable, however, that some plants classed as crinkly do not show prominently one or other of them. Considering all these leaf characters together with stature and form of tassel, it is usually easily possible to separate crinkly from normal plants, but occasionally the separation is somewhat difficult.

In stocks of crinkly in which the characters noted above are developed to an extreme degree the tassels ordinarily have great difficulty in pushing out from the more or less rolled upper leaves (Fig. 26). It is sometimes necessary to slit the upper leaves to release the tassels and allow the pollen to be shed normally.

The ears of crinkly are usually somewhat smaller than those of normal plants but are not otherwise materially different.

\section{INHERITANCE OF CRINKLY}

Crosses of crinkly with normal have invariably given normal $F_{1}$ plants. Self-pollinated $F_{1}$ plants produced 717 $\mathrm{F}_{2}$ 's of which 544 were normal and 173 crinkly, a deviation of 6.3 I 7.8 from the $3: 1$ ratio. From backcrosses of $F_{1}$ normals with crinkly a total of 3,567 plants resulted. Of these 1,850 were normal and 1,717 crinkly. This is a deviation of 66.5 I 20.1 from equality. So great a deviation could be expected by chance alone only about once in 38 trials. In some of the backcross progenies the crinkly plants were not so easily separated from normals as in others. It seems likely that this difficulty in identifying crinkly in some cultures may explain in part the deficiency of that type, but it is possible also that crinkly does not survive under crowded field conditions as well as normal plants. Most dwarf and semi-dwarf types of maize, such as dwarf, anther ear, tassel ear, etc., almost always are deficient in field cultures, particularly when grown under unfavorable conditions, and there is no apparent reason to expect crinkly to behave very differently from some of these other types.

Self-pollinated crinkly plants have invariably produced nothing but crinkly progeny. Something over one hundred crinkly plants have been observed in such cultures.

1 Paper No. 92, Department of Plant Breeding, Cornell University, Ithaca, New York. 



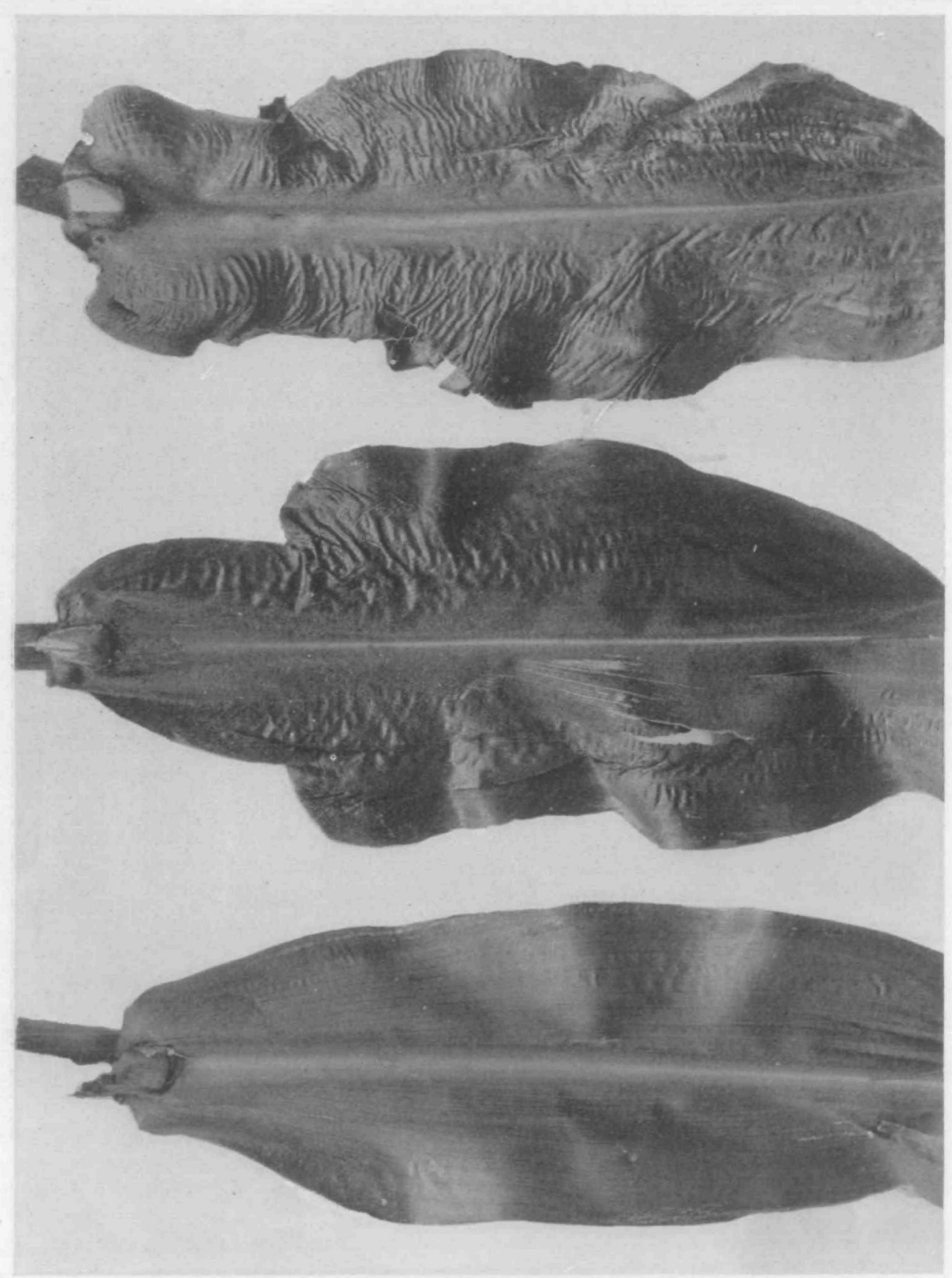

\section{CRINKLY AND NORMAL MAIZE LEAVES}

The leaves of Crinkly are not only crinkled but often have prominent lobes near the base of the blades. These leaf characteristics are variable, however, and although it is usually easy to distinguish crinkly from normal plants, separation is occasionally somewhat difficult. (Fig. 25.) 


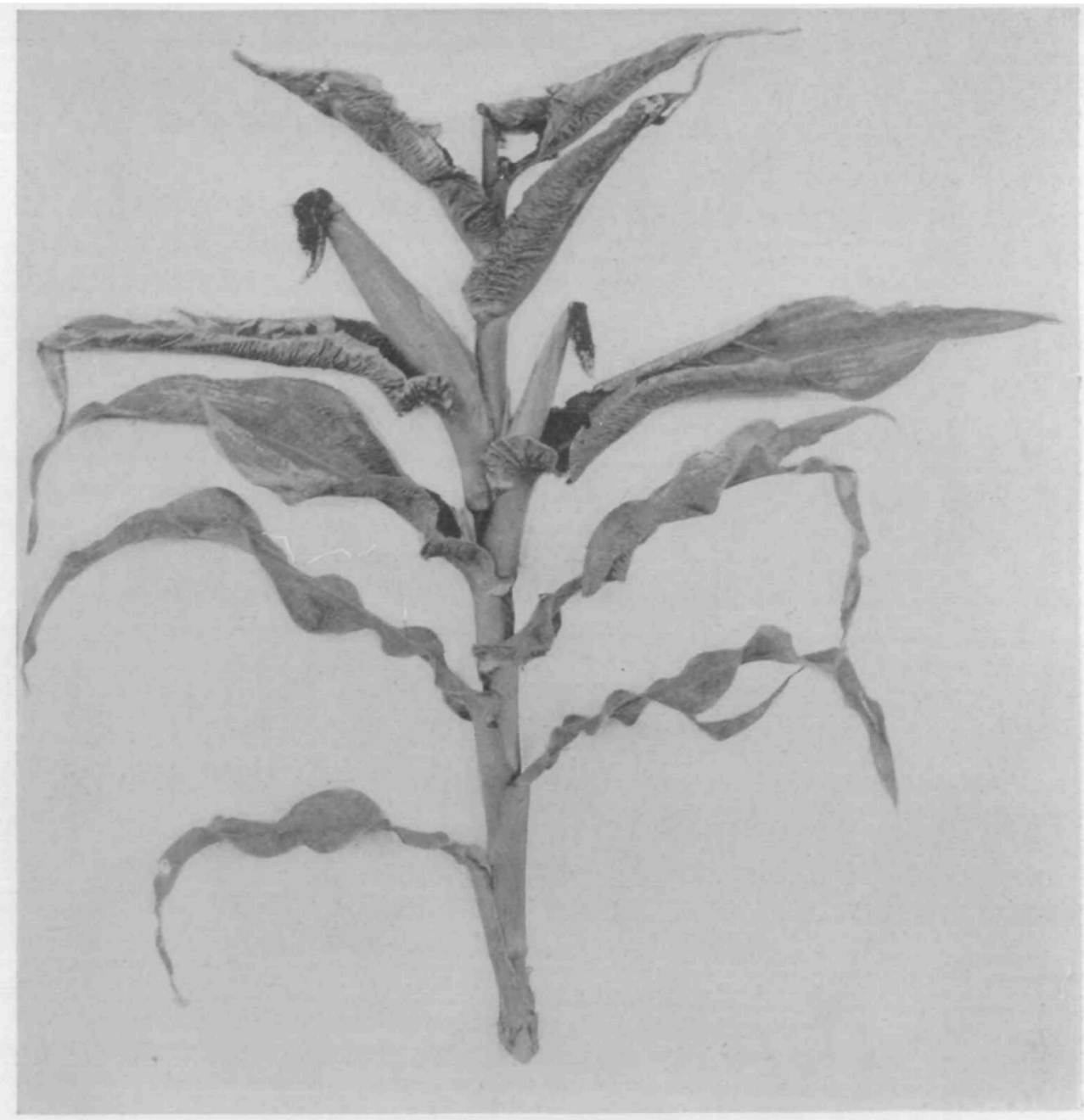

\section{AN EXTREME TYPE OF CRINKLY MAIZE}

In some stocks of Crinkly, especially those in which the abnormal characteristics are developed to an extreme degree, the tassels have difficulty in pushing out of the rolled upper leaves. It is sometimes necessary to slit the upper leaves. "The ears of crinkly are usually somewhat smaller than those of normal plants but are not otherwise materially different." (Fig. 26.)

On the whole, it is thought safe to conclude that crinkly is a simple mendelian recessive to normal and that the two types are differentiated by the single factor pair $\mathrm{Cr} \mathrm{cr}$. That the expression of the character crinkly may be influenced by modifying factors seems likely, however, in view of the noticeable differences in height of plants and in breadth, crinkliness, and lobing of leaves in different stocks. 